\title{
Spiro Diorthoester (SpiDo), a Human Plasma Stable Acid-Sensitive Cleavable Linker for Lysosomal Release
}

\author{
Geoffray Leriche, ${ }^{\dagger}$ Marc Nothisen, ${ }^{\ddagger}$ Nadège Baumlin, ${ }^{\S}$ Christian D. Muller,, Dominique Bagnard, ${ }^{\S}$ \\ Jean-Serge Remy, ${ }^{\dagger}$ Sylvain A. Jacques, ${ }^{*}{ }^{\dagger}$ and Alain Wagner ${ }^{*}{ }^{\dagger}$ \\ ${ }^{\dagger}$ LFCS and ${ }^{\ddagger}$ V-SAT laboratories, CAMB UMR 7199 CNRS University of Strasbourg, LabEx Medalis, icFRC, Faculty of Pharmacy, 74 \\ route du Rhin, 67400 Illkirch, France \\ ${ }^{\S}$ INSERM U1109 - MN3T Lab, University of Strasbourg, LabEx Medalis, Fédération de Médecine Translationnelle de Strasbourg \\ (FMTS), 67000 Strasbourg, France \\ "Laboratoire d'Innovation Thérapeutique, UMR 7200, CNRS University of Strasbourg, Faculty of Pharmacy, 74 route du Rhin, 67400 \\ Illkirch, France
}

\section{Supporting Information}

\begin{abstract}
H}$-sensitive linkers designed to undergo selective hydrolysis at acidic $\mathrm{pH}$ compared to physiological $\mathrm{pH}$ can be used for selective release of therapeutics selectively at targets and orthoesters have been demonstrated to be good candidates for such linkers. Following an HPLC screening, a Spiro Diorthoester (SpiDo) derivative was identified as a potent acid-labile group for the development of $\mathrm{pH}$-sensitive targeted systems. After incorporation of this linker into activatable FRET-based probe and side-by-side comparison to a well-known alkylhydrazone linker, this SpiDo linker has shown a fast and $\mathrm{pH}$ sensitive hydrolysis for mild acidic conditions, a $\mathrm{pH}$ sensitive lysosomal hydrolysis, and high stability in human plasma.
\end{abstract}

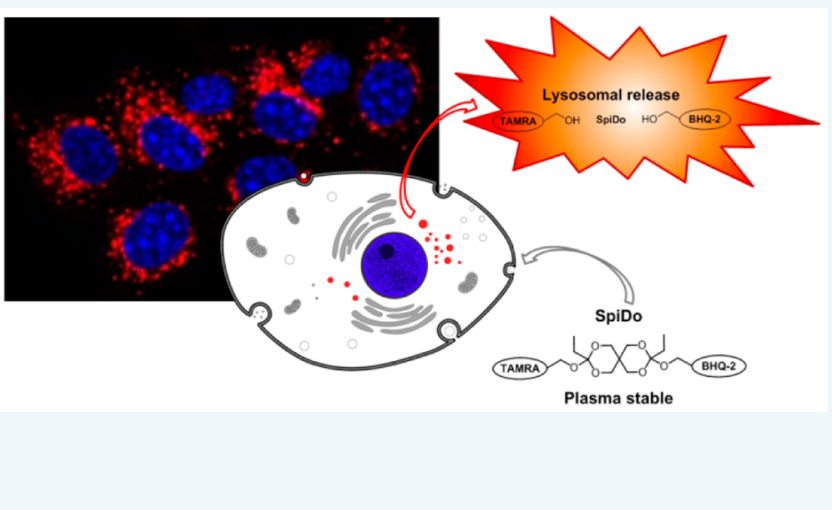

\section{INTRODUCTION}

In cancer therapy, many successful strategies to increase the therapeutic index of highly cytotoxic drug involve prodrugs that release the active drug once it has reached its target. ${ }^{1}$ Such an approach relies on the ability of a linker to be cleaved in response to physiological, pathological, or pathochemical conditions (e.g., enzyme overexpression, redox microenvironment, and $\mathrm{pH}$ gradient). ${ }^{2,3}$ Acidic $\mathrm{pH}$-gradients have been extensively used as biological stimuli to trigger the release of delivery systems and, as a result of many studies, several acidlabile structures have been reported over time in the design of drug delivery systems, via protonation mechanisms such as $\mathrm{pH}$ dependent microcapsules ${ }^{4}$ or bond breaking mechanisms in polymers $^{5}$ and molecular entities (e.g., acylhydrazone, ${ }^{6-9}$ acetal, $^{10}$ silyl ether, ${ }^{11,12}$ cis-aconityl, ${ }^{13} \beta$-thiopropionate, ${ }^{14,15}$ thiomaleamic, ${ }^{16}$ and imidazole ${ }^{17,18}$ derivatives). These chemical groups have been used for both intra- and extracellular drug release. Upon cellular internalization, a delivery system undergoes passage through acidic endosomal/lysosomal compartments, at $\mathrm{pH} \mathrm{6-6.8} \mathrm{and} \mathrm{4.5-5.5,} \mathrm{respectively,} \mathrm{cleaving}$ the labile linker and releasing the drug payload. ${ }^{19}$ Alternatively, the extracellular hypoxic tumor microenvironment is reported to be $0.5-1 \mathrm{pH}$ unit more acidic than healthy tissue and blood.

Most of the industrial approaches for antibody-drug conjugates $(\mathrm{ADC})$ encompassed the use of hydrazone linkers which have been used to facilitate the release of a highly toxic payload after ADC internalization (Mylotarg and Inotuzumab ozogamicin). ${ }^{20}$ However, both Myoltarg and Inotuzumab ozogamicin have been withdrawn from the market and phase III clinical trial. The withdrawal of Mylotarg was attributed to toxicities related to acylhydrazone linker instability, which resulted in an increased number of treatment-related mortalities. $^{21}$ In the course of bridging the gap between shielding normal tissue from toxic compounds and delivering drugs at therapeutic concentrations to target cells, we have explored the use of a Spiro Diorthoester (SpiDo) for lysosomal release. Heller $^{22-28}$ and Szoka ${ }^{29-32}$ have already prepared a series of polymers based on the diorthoester moiety and exploited their $\mathrm{pH}$-sensitive properties to develop sustained drug release systems. Of particular importance, the half-life of liposomal formulations of Spiro Diorthoester were reported by Szoka and Guo $^{29,30}$ to be approximately $2.5 \mathrm{~h}$ at $\mathrm{pH} 6$, and in vivo experiments showed that the lipid derivatives can be used to further modify the delivery properties of the lipidic delivery system in order to enable prolonged circulation time. (e.g., by changing the lipid chain and the polar head). All of these

Received: May 18, 2015

Revised: June 30, 2015

Published: July 1, 2015 
Scheme 1. Synthesis of Probes 1-3
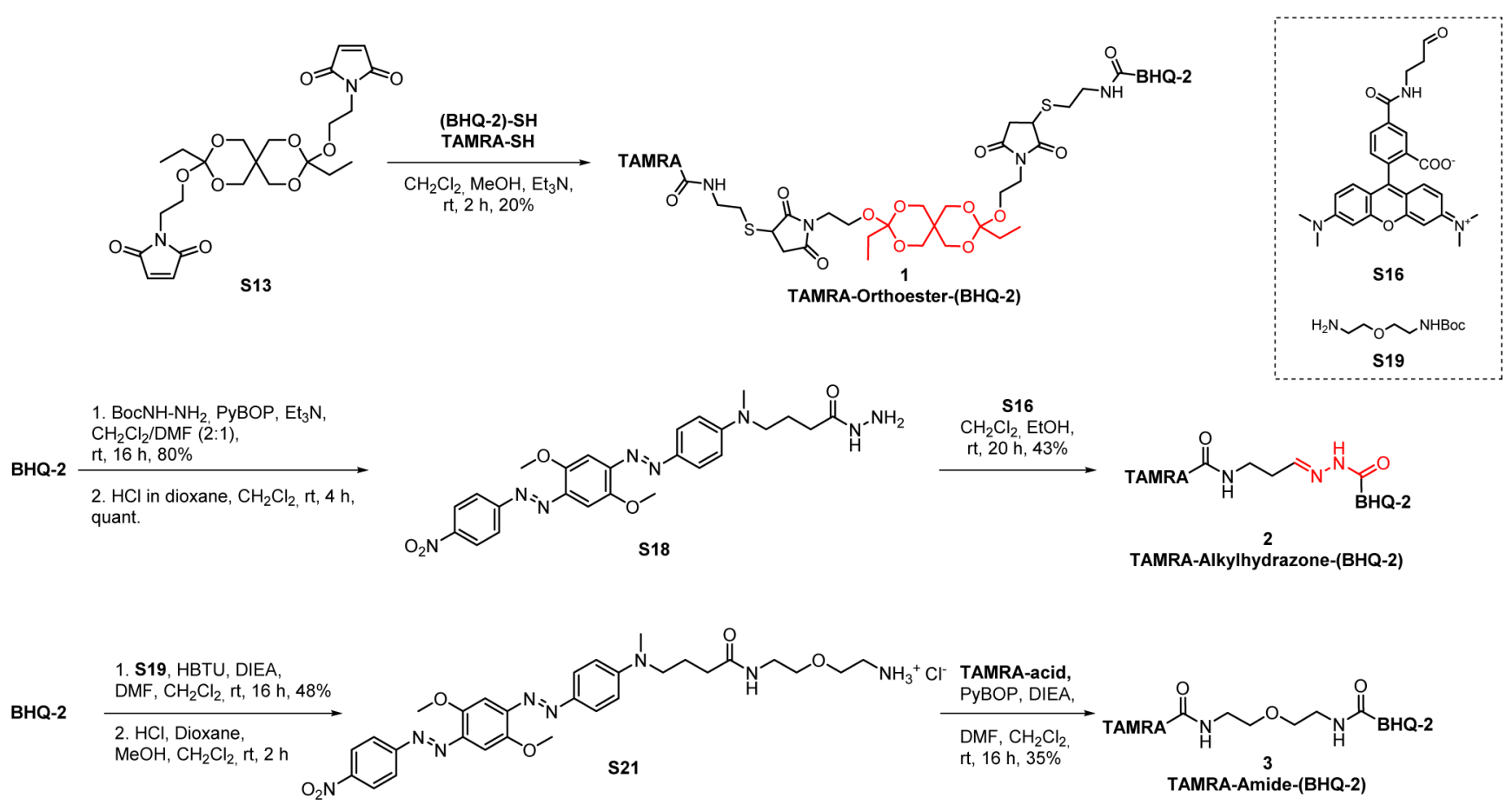

literature reports revealed the high potency of orthoesters in the field of bioresponsive polymers and acid-labile liposomes. However, to the best of our knowledge, the precise behavior of the Spiro Diorthoester in response to specific biological stimuli and in the response induced by lysosomal uptake has not been reported on its own. Consequently, its potency to be used as a cleavable linker in targeted systems such as ADCs, which requires both long plasma stability and fast lysosomal cleavage, has not yet been clearly established. The aim of this study is to establish and demonstrate this potency by comparing with the same method, the hydrolysis profile and reactivity of this specific linker to the commonly used acylhydrazone linker. Albeit other $\mathrm{pH}$ sensitive groups such as acetals are already known, we decided to solely compare the SpiDo linker to the well-known acylhydrazone linker.

\section{RESULTS AND DISCUSSION}

Literature analyses show that various acid-sensitive linkers have been developed to date. However, the difference in experimental condition (buffer, temperature, concentration, etc.) between the different studies makes direct comparison of stability and degradability of different linkers very difficult. To address this problem, we have conducted within our group an HPLC-based hydrolysis assay and recorded the hydrolysis profiles of a broad selection of acid-sensitive groups regularly used in organic chemistry, i.e., acylhydrazone, acetal, dialkylacetal, aryl-1,3-dioxolane, aryl-1,3-dioxane, ethoxybenzylimidazole, oxime, imine, and 3-alkylthiopropionate moieties. From this study, we have identified a Spiro Diorthoester (SpiDo) structure $\mathbf{S} 3$ which displayed a rapid hydrolysis profile selective toward acidic conditions ( $\mathrm{pH}$ 5.5) relative to physiological $\mathrm{pH}$ (see SI Figure S2). For comparison, we prepared acyl and alkyl hydrazones derivatives (S4-S7) (see SI Figure S3) and compared their hydrolysis profiles (see SI Figure S4). Whereas acylhydrazones S4 and S5 showed little hydrolysis at pH 5.5 and 7.4, alkylhydrazones S6 and S7 were more sensitive to acidic conditions, and the most stable alkylhydrazone S6 was chosen for comparison with S3.

With this promising result in hand, we then proceeded to further compare the biosensitivities of SpiDo and alkylhydrazone. To ensure a rapid and efficient method for measuring hydrolysis in vitro, the SpiDo and alkylhydrazone linkers were incorporated into activatable FRET-based probes $\mathbf{1}$ and 2, respectively, whose 5-carboxytetramethylrhodamine (TAMRA) fluorescence is quenched by Black Hole Quencher-2 dye (BHQ-2) via intramolecular FRET. Upon acid-catalyzed hydrolysis of the $\mathrm{pH}$-sensitive linker, the TAMRA and BHQ2 are separated and the fluorescence of TAMRA can be detected. A FRET-based probe 3 without $\mathrm{pH}$-sensitive linker has also been synthesized as a negative control. Of particular interest, we designed the FRET-based TAMRA-OrthoesterBHQ-2 probe $\mathbf{1}$ on the basis of a previously reported bismaleimide spiro diorthoester used for protein cross-linking. ${ }^{33}$ Starting from already known bis-maleimide Spiro Diorthoester $\mathrm{S13}^{33}$ probe 1 was synthesized in one step by simultaneous treatment of 4 with (BHQ-2)-SH ${ }^{34}$ and TAMRA-SH ${ }^{34}$ in $20 \%$ yield (Scheme 1). Probe 2 was synthesized in three steps starting from BHQ-2. Coupling of BHQ-2 with mono-Boc protected hydrazine followed by deprotection with $\mathrm{HCl}$ gave hydrazide S18, which was further condensed with freshly prepared aldehyde $\mathrm{S16}^{34}$ to give probe 2 in average yield. Finally, probe 3 was prepared in 3 steps starting from BHQ-2. Coupling of BHQ-2 with synthesized linker S19, ${ }^{34}$ followed by BOC deprotection using $\mathrm{HCl}$, gave $\mathbf{S 2 1}$ which was further coupled with TAMRA-acid using PyBOP to give probe 3 . The synthesis of probes 1 and 2 required careful attention with the use of mild conditions when handling the highly $\mathrm{pH}$-sensitive group, and at the final stage of the synthesis, the probes were purified by preparative HPLC using $\mathrm{NH}_{3} / \mathrm{HCOOH}$ solution (10 mM, pH 8.5)/MeCN as eluent system and no degradation was observed. 
Initially, kinetic measurements based on molecular fluorescence were conducted to evaluate the stability of the different probes for $15 \mathrm{~h}$ in aqueous buffer solutions at $\mathrm{pH} 5.5$ (to simulate the lysosomal $\mathrm{pH}$ ) and $\mathrm{pH} 7.4$ (to simulate serum conditions). At $\mathrm{pH} 5.5$, probe 1 showed a rapid activation of fluorescence due to the hydrolysis of the SpiDo linker (Figure 1A). Probe 1 displayed a slight increase of fluorescence at $\mathrm{pH}$

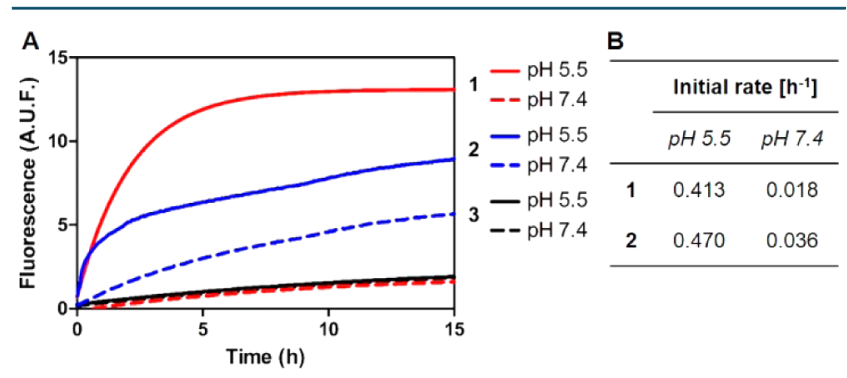

Figure 1. Hydrolysis kinetics of FRET-based probes 1-3. (A) Hydrolysis kinetics of FRET-based probes 1 (red lines), 2 (blue lines), and 3 (black lines) at pH 5.5 (solid lines) and 7.4 (dashed lines). All data were recorded in triplicate at room temperature on a microplate reader and with a probe concentration of $0.5 \mu \mathrm{M}$ in phosphate buffers $\left(\mathrm{NaH}_{2} \mathrm{PO}_{4} / \mathrm{Na}_{2} \mathrm{HPO}_{4} \mathrm{pH} 7.4\right.$ or $\mathrm{KH}_{2} \mathrm{PO}_{4} / \mathrm{Na}_{2} \mathrm{HPO}_{4} \mathrm{pH} 5.5,100$ $\mathrm{mM}$ ). (B) Initial rate kinetics calculated for $10 \%$ of conversion of 1 and 2 at $\mathrm{pH} 5.5$ and 7.4 assuming a first-order reaction.

7.4 but comparable to nonhydrolyzable probe 3 . Initial rate kinetics were compared and indicated that the hydrolysis of $\mathbf{1}$ in acidic conditions was 23 -fold faster than at physiological $\mathrm{pH}$ (Figure 1B). The hydrolysis half time was found to be $1.5 \mathrm{~h}$ (assuming a first-order reaction) and full hydrolysis was completed after $7 \mathrm{~h}$ at $\mathrm{pH}$ 5.5.

Under the same experimental conditions as with probe 1, alkylhydrazone probe $\mathbf{2}$ displayed completely different $\mathrm{pH}$ dependent hydrolysis profiles as well as reaction equilibration (Figure 1A). Two different rates were established: (i) a firstorder reaction occurring within the first hour associated with a burst release and (ii) a slower rate, which was attributed to an equilibrium state due to the reversible nature of hydrolysis of the alkylhydrazone. ${ }^{35}$ Running the same experiment with an excess of nucleophilic hydrazine to induce transamination reaction and eliminate the reversibility of alkylhydrazone hydrolysis confirmed the equilibrated reaction (see SI Figure S8). At $\mathrm{pH} 7.4$, significant hydrolysis of probe 2 was observed compared to control probe 3 . Kinetic data analysis revealed that hydrolysis of 2 is 13 -fold faster in acidic conditions. Interestingly, while initial rates of $\mathbf{1}$ and $\mathbf{2}$ appear to be quite similar at $\mathrm{pH} 5.5,1$ was considerably more stable at $\mathrm{pH} 7.4$ suggesting that $\mathrm{SpiDo}$ is more sensitive to $\mathrm{pH}$-variation than the alkylhydrazone linker (Figure 1B).

The biosensitivities of the different probes $(1-3)$ were also studied in tissue cultures and cells were analyzed by confocal microscopy as well as flow cytometry to provide spatial and quantitative information about intracellular probe hydrolysis. First, BNL CL.2, a mouse liver cell line, was loaded with the various probes $(1 \mu \mathrm{M}, 90 \mathrm{~min})$ with nuclei stained by Hoechst 33258. Representative images are shown in Figure 2 (images A-C). SpiDo-based probe 1 exhibited the expected fluorescent signal with a vesicular distribution (image A) compared to negative control 3 (image $\mathrm{C}$ ). In the case of alkylhydrazonebased probe 2, fluorescence activation was also observed, however, with lower intensities (Figure 2B). In order to associate fluorescence activation to intracellular acidic con-
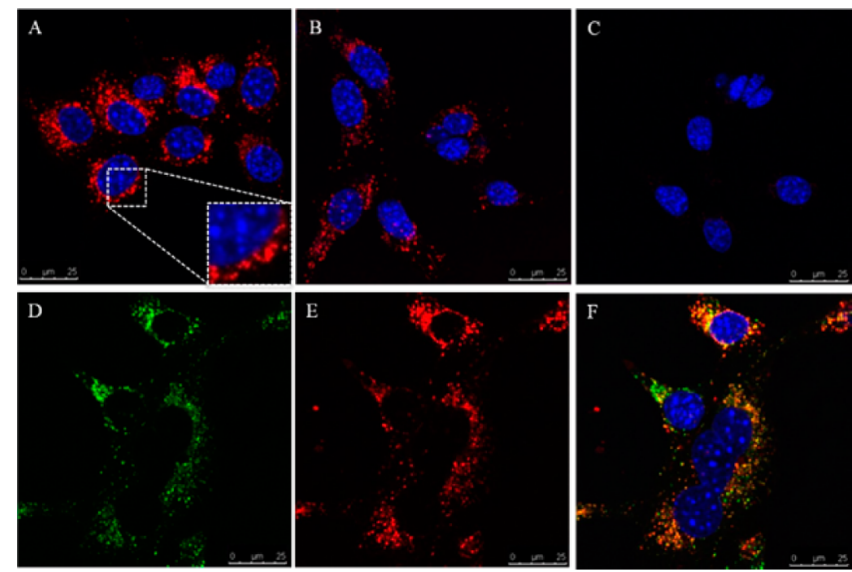

Figure 2. In vitro imaging of BNL CL.2 cells loaded with probes 1-3. In vitro imaging of BNL CL. 2 cells loaded with probes 1 (image A), 2 (image B), and 3 (image C). Distribution and colocalization of $\mathbf{1}$ with lysosomal probe DND-26 (images D-F). Image D: green channel = lysotracker DND-26 fluorescence, image $\mathrm{E}$ : red channel $=\mathbf{1}$ fluorescence, image F: merge channels. Live cell staining and imaging were carried out by initially staining with the different probes $(1 \mu \mathrm{M}$, $90 \mathrm{~min}$ ) followed by staining with Hoechst 33258 to stain the nuclei $(5 \mu \mathrm{g} / \mathrm{mL}, 30 \mathrm{~min})$. For colocalization experiments, cells were stained with LysoTracker Green DND-26 (100 nM, $30 \mathrm{~min})$. Confocal fluorescence microscopy of living cells was performed. Scale bar: 25 $\mu \mathrm{m}$.

ditions, 1 was costained with LysoTracker Green DND-26, which is known to stain acidic compartments in live cells such as lysosomes. To our delight, cells displayed colocalization of the red and green fluorescence as a result of the TAMRA and lysotracker, respectively, resulting in an orange signal in a merged picture (images $\mathrm{D}-\mathrm{F}$ ). This colocalization suggests that the probe is taken up by cells to be specifically hydrolyzed in acidic organelles. Moreover, flow cytometry experiments were conducted for additional quantitative comparison. Cells were incubated with probes 1 and 2 for $4 \mathrm{~h}$, suspended in media and analyzed. Probe 1 exhibited a 2.6-fold higher level of fluorescence (A.U.F.) than probe 2, confirming an increased rate of hydrolysis of SpiDo relative to alkylhydrazone (see SI Figure S9). These cellular results are in good agreement with buffer based experiments (Figure 1).

To further validate that hydrolysis of $\mathbf{1}$ is triggered by $\mathrm{pH}$ and not by any other mechanism, endosomal/lysosomal system acidification was inhibited by chloroquine (CQ) which is a weak base known to quickly raise intracellular $\mathrm{pH} .{ }^{36}$ Cells were pretreated with chloroquine $(100 \mu \mathrm{M}, 2 \mathrm{~h})$ prior to probe incubation $(1 \mu \mathrm{M}, 4 \mathrm{~h})$, and analyzed by flow cytometry. Fluorescence activation in CQ-treated cells considerably decreased by $45 \%$ and $35 \%$ for 1 and 2, respectively, compared to untreated cells (Figure 3 and SI Figure S10). These results clearly demonstrate that activation of both 1 and $\mathbf{2}$ occurred intracellularly due to $\mathrm{pH}$ variation. The lower effect of CQtreatment on probe $\mathbf{2}$ may suggest other mechanisms for its activation, such as transamination reactions with proteins. Finally, we decided to investigate the stabilities of probes 1-3 in human plasma (Figure 4). A strong increase of fluorescence was observed for alkylhydrazone-based probe 2 , while the level of fluorescence of SpiDo probe $\mathbf{1}$ remained at a level comparable to control probe 3 . 


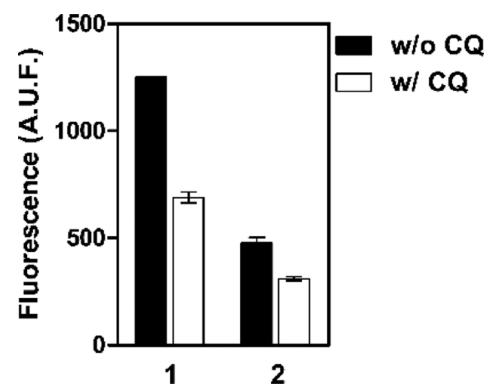

Figure 3. Flow cytometry analysis of probes 1-2. Flow cytometry analysis (2000 cells/assay) of BNL CL.2 cells loaded with probes 1 and $2(1 \mu \mathrm{M}, 4 \mathrm{~h})$ with (white bars) or without (black bars) CQpretreatment $(100 \mu \mathrm{M}, 2 \mathrm{~h})$.

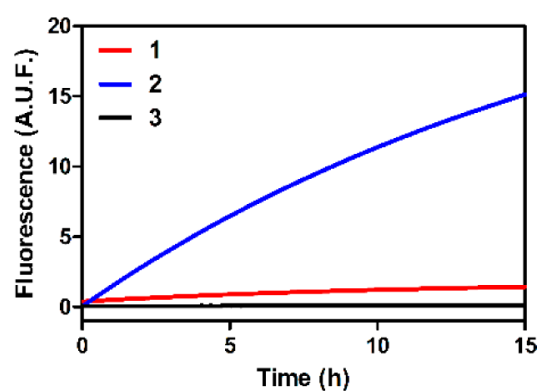

Figure 4. Hydrolysis kinetics of FRET-based probes 1-3 in Human plasma. Hydrolysis kinetics of FRET-based probes 1 (red line), 2 (blue line), and 3 (black line) in Human plasma (50\% in PBS). All data were measured in triplicate at $37{ }^{\circ} \mathrm{C}$ on a microplate reader and with a probe concentration of $0.5 \mu \mathrm{M}$.

\section{CONCLUSION}

In conclusion, we have revealed that Spiro Diorthoester (SpiDo) derivative is a potent acid-labile group for the development of $\mathrm{pH}$-sensitive targeted systems. After HPLCbased kinetics of model compounds, the SpiDo motif was inserted into FRET-based probes and the hydrolysis kinetics was recorded and compared to a related acylhydrazone FRETbased probe. This specific SpiDo linker has shown a fast and $\mathrm{pH}$ sensitive hydrolysis under mild acidic conditions. The biosensitivities of the different probes were also studied in cellular systems and colocalization experiments showed that the SpiDo FRET-based probe was specifically hydrolyzed in acidic organelles. Finally, stability of both probes was investigated in human plasma and the SpiDo probe proved to be highly stable compared to the acylhydrazone probe. As a result, the profile of SpiDo makes it a very promising acid-labile group for $\mathrm{pH}$ sensitive targeted systems such as internalizing ADCs.

\section{ASSOCIATED CONTENT}

\section{S Supporting Information}

Complete experimental procedures, characterization of new compounds. The Supporting Information is available free of charge on the ACS Publications website at DOI: 10.1021/ acs.bioconjchem.5b00280.

\section{AUTHOR INFORMATION}

\section{Corresponding Authors}

*E-mail: s.jacques@unistra.fr.

*E-mail: alwag@unistra.fr.

\section{Notes}

The authors declare no competing financial interest.

\section{ACKNOWLEDGMENTS}

This work was supported through a research contract funded by the French Ministry of Research. The International Center for Frontier Research in Chemistry (icFRC) is acknowledged for financial support to LFCS.

\section{REFERENCES}

(1) Bildstein, L., Dubernet, C., and Couvreur, P. (2011) Prodrugbased intracellular delivery of anticancer agents. Adv. Drug Delivery Rev. 63, 3-23.

(2) Leriche, G., Chisholm, L., and Wagner, A. (2012) Cleavable linkers in chemical biology. Bioorg. Med. Chem. 20, 571-582.

(3) Ganta, S., Devalapally, H., Shahiwala, A., and Amiji, M. (2008) A review of stimuli-responsive nanocarriers for drug and gene delivery. J. Controlled Release 126, 187-204.

(4) Mauser, T., Déjugnat, C., and Sukhorukov, G. B. (2004) Reversible pH-Dependent Properties of Multilayer Microcapsules Made of Weak Polyelectrolytes. Macromol. Rapid Commun. 25, 17811785.

(5) Delcea, M., Möhwald, H., and Skirtach, A. G. (2011) Stimuliresponsive LbL capsules and nanoshells for drug delivery. Adv. Drug Delivery Rev. 63, 730-747.

(6) Bae, Y., Jang, W.-D., Nishiyama, N., Fukushima, S., and Kataoka, K. (2005) Multifunctional polymeric micelles with folate-mediated cancer cell targeting and $\mathrm{pH}$-triggered drug releasing properties for active intracellular drug delivery. Mol. BioSyst. 1, 242-250.

(7) Bae, Y., Nishiyama, N., and Kataoka, K. (2007) In Vivo Antitumor Activity of the Folate-Conjugated $\mathrm{pH}$-Sensitive Polymeric Micelle Selectively Releasing Adriamycin in the Intracellular Acidic Compartments. Bioconjugate Chem. 18, 1131-1139.

(8) Kaneko, T., Willner, D., Monkovic, I., Knipe, J. O., Braslawsky, G. R. Greenfield, R. S., and Vyas, D. M. (1991) New hydrazone derivatives of Adriamycin and their immunoconjugates - a correlation between acid stability and cytotoxicity. Bioconjugate Chem. 2, 133-141.

(9) Biswas, S., Dodwadkar, N. S., Sawant, R. R., and Torchilin, V. P. (2011) Development of the Novel PEG-PE-Based Polymer for the Reversible Attachment of Specific Ligands to Liposomes: Synthesis and in Vitro Characterization. Bioconjugate Chem. 22, 2005-2013.

(10) Liu, R, Zhang, Y., Zhao, X., Agarwal, A., Mueller, L. J., and Feng, P. (2010) pH-Responsive Nanogated Ensemble Based on GoldCapped Mesoporous Silica through an Acid-Labile Acetal Linker. J. Am. Chem. Soc. 132, 1500-1501.

(11) Sankaranarayanan, J., Mahmoud, E. A., Kim, G., Morachis, J. M., and Almutairi, A. (2010) Multiresponse Strategies To Modulate Burst Degradation and Release from Nanoparticles. ACS Nano 4, 59305936.

(12) Parrott, M. C., Luft, J. C., Byrne, J. B., Fain, J. H., Napier, M. E., and DeSimone, J. M. (2010) Tunable Bifunctional Silyl Ether CrossLinkers for the Design of Acid Sensitive Biomaterials. J. Am. Chem. Soc. $132,17928-17932$.

(13) Shen, W. C., and Ryser, H. J. P. (1981) cis-Aconityl spacer between daunomycin and macromolecular carriers: a model of $\mathrm{pH}$ sensitive linkage releasing drug from a lysosomotropic conjugate. Biochem. Biophys. Res. Commun. 102, 1048-1054.

(14) Oishi, M., Sasaki, S., Nagasaki, Y., and Kataoka, K. (2003) pHResponsive Oligodeoxynucleotide (ODN)-Poly(Ethylene Glycol) Conjugate through Acid-Labile $\beta$-Thiopropionate Linkage: Preparation and Polyion Complex Micelle Formation. Biomacromolecules 4, 1426-1432.

(15) Oishi, M., Nagasaki, Y., Itaka, K., Nishiyama, N., and Kataoka, K. (2005) Lactosylated Poly(ethylene glycol)-siRNA Conjugate through Acid-Labile $\beta$-Thiopropionate Linkage to Construct $\mathrm{pH}$-Sensitive Polyion Complex Micelles Achieving Enhanced Gene Silencing in Hepatoma Cells. J. Am. Chem. Soc. 127, 1624-1625.

(16) Castañeda, L., Maruani, A., Schumacher, F. F., Miranda, E., Chudasama, V., Chester, K. A., Baker, J. R., Smith, M. E. B., and Caddick, S. (2013) Acid-cleavable thiomaleamic acid linker for 
homogeneous antibody-drug conjugation. Chem. Commun. 49, 81878189.

(17) Kong, S. D., Luong, A., Manorek, G., Howell, S. B., and Yang, J. (2007) Acidic Hydrolysis of N-Ethoxybenzylimidazoles (NEBIs): Potential Applications as $\mathrm{pH}$-Sensitive Linkers for Drug Delivery. Bioconjugate Chem. 18, 293-296.

(18) Luong, A., Issarapanichkit, T., Kong, S. D., Fong, R., and Yang, J. (2010) pH-Sensitive, $N$-ethoxybenzylimidazole (NEBI) bifunctional crosslinkers enable triggered release of therapeutics from drug delivery carriers. Org. Biomol. Chem. 8, 5105-5109.

(19) Urano, Y., Asanuma, D., Hama, Y., Koyama, Y., Barrett, T., Kamiya, M., Nagano, T., Watanabe, T., Hasegawa, A., Choyke, P. L., and Kobayashi, H. (2009) Selective molecular imaging of viable cancer cells with $\mathrm{pH}$-activatable fluorescence probes. Nat. Med. 15, 104-109.

(20) Sapra, P., Hooper, A. T., O’Donnell, C. J., and Gerber, H.-P. (2011) Investigational antibody drug conjugates for solid tumors. Expert Opin. Invest. Drugs 20, 1131-1149.

(21) Perez, H. L., Cardarelli, P. M., Deshpande, S., Gangwar, S., Schroeder, G. M., Vite, G. D., and Borzilleri, R. M. (2014) Antibodydrug conjugates: current status and future directions. Drug Discovery Today 19, 869-881.

(22) Heller, J. (1985) Controlled Drug Release from Poly(ortho esters). Ann. N. Y. Acad. Sci. 446, 51-66.

(23) Merkli, A., Heller, J., Tabatabay, C., and Gurny, R. (1993) Synthesis and characterization of a new biodegradable semi-solid poly(ortho ester) for drug delivery systems. J. Biomater. Sci., Polym. Ed. $4,505-16$.

(24) Toncheva, V., Schacht, E., Ng, S. Y., Barr, J., and Heller, J. (2003) Use of Block Copolymers of Poly(Ortho Esters) and Poly (Ethylene Glycol) Micellar Carriers as Potential Tumour Targeting Systems. J. Drug Target. 11, 345-353.

(25) Heller, J. (1990) Development of poly(ortho esters): a historical overview. Biomaterials 11, 659-665 and references herein. .

(26) Heller, J., and Barr, J. (2004) Poly(ortho esters) From Concept to Reality. Biomacromolecules 5, 1625-1632.

(27) Heller, J., Barr, J., Ng, S. Y., Schwach Abdellauoi, K., and Gurny, R. (2002) Poly(ortho esters): synthesis, characterization, properties and uses. Adv. Drug Delivery Rev. 54, 1015-1039.

(28) Sintzel, M. B., Heller, J., Ng, S. Y., Tabatabay, C., SchwachAbdellaoui, K., and Gurny, R. (1998) In vitro drug release from selfcatalyzed poly(ortho ester): Case study of 5-fluorouracil. J. Controlled Release 55, 213-218.

(29) Guo, X., and Szoka, F. C., Jr. (2001) Steric stabilization of fusogenic liposomes by a low-pH sensitive PEG-Diortho ester-lipid conjugate. Bioconjugate Chem. 12, 291-300.

(30) Guo, X., and Szoka, F. C., Jr. (2005) pH sensitive lipids based on orthoester linkers, compositions and method. US 6,897,196 B1.

(31) Huang, Z., Guo, X., MacKay, J. A., and Szoka, F. C., Jr. (2006) Acid-Triggered Transformation of Diortho Ester Phosphocholine Liposome. J. Am. Chem. Soc. 128, 60-61.

(32) Guo, X., MacKay, J. A., and Szoka, F. C., Jr. (2003) Mechanism of $\mathrm{pH}$-triggered collapse of phosphatidyl ethanolamine liposomes stabilized by an ortho ester polyethylene glycol lipid. Biophys. J. 84, 1784-1795.

(33) Srinivasachar, K., and Neville, D. M. (1989) New protein crosslinking reagents that are cleaved by mild acid. Biochemistry 28, 2501-2509.

(34) For a detailed synthesis of (BHQ-2)-SH S9, TAMRA-SH S11, aldehyde S16 and linker S19, see Supporting Information.

(35) Kalia, J., and Raines, R. T. (2008) Hydrolytic stability of hydrazones and oximes. Angew. Chem., Int. Ed. 47, 7523-7526.

(36) Kaiden, T., Yuba, E., Harada, A., Sakanishi, Y., and Kono, K. (2011) Dual signal-responsive liposomes for temperature-controlled cytoplasmic delivery. Bioconjugate Chem. 22, 1909-1915. 